\title{
Electron and hole mobility reduction and Hall factor in phosphorus-compensated p-type silicon
}

\author{
F. E. Rougieux, ${ }^{1, a)}$ D. Macdonald, ${ }^{1}$ A. Cuevas, ${ }^{1}$ S. Ruffell, ${ }^{2}$ J. Schmidt, ${ }^{3}$ B. Lim, ${ }^{3}$ and \\ A. P. Knights ${ }^{4}$ \\ ${ }^{1}$ School of Engineering, College of Engineering and Computer Science, The Australian National University, \\ Canberra ACT 0200, Australia \\ ${ }^{2}$ Department of Electronic Materials Engineering, Research School of Physics and Engineering, The \\ Australian National University, Canberra ACT 0200, Australia \\ ${ }^{3}$ Institut für Solarenergieforschung Hameln (ISFH), Am Ohrberg 1, D-31860 Emmerthal, Germany \\ ${ }^{4}$ McMaster University, 1280 Main Street West, Hamilton, Ontario L8S 4L7, Canada
}

(Received 15 April 2010; accepted 21 May 2010; published online 7 July 2010)

\begin{abstract}
The conductivity mobility for majority carrier holes in compensated $p$-type silicon is determined by combined measurement of the resistivity and the net doping, the latter via electrochemical capacitance-voltage measurements. The minority electron mobility was also measured with a technique based on measurements of surface-limited effective carrier lifetimes. While both minority and majority carrier mobilities are found to be significantly reduced by compensation, the impact is greater on the minority electron mobility. The Hall factor, which relates the Hall mobility to the conductivity mobility, has also been determined using the Hall method combined with the capacitance-voltage measurements. Our results indicate a similar Hall factor in both compensated and noncompensated samples. (C) 2010 American Institute of Physics. [doi:10.1063/1.3456076]
\end{abstract}

\section{INTRODUCTION}

Charge carrier mobilities are limited by the amount of scattering taking place in a semiconductor. The scattering mechanisms are mainly lattice scattering, carrier-carrier scattering, and defect scattering (crystal defects and impurities). Both ionized and neutral impurities can influence the mobility but in relatively pure monocrystalline silicon at room temperature, ionized impurity, carrier-carrier, and lattice scattering dominate. A large amount of experimental data and numerous models concerning mobilities in noncompensated silicon exist in the literature. ${ }^{1-9}$ This allows one to easily deduce the bulk dopant density from resistivity data, for example. Knowing the dopant density one can also predict the minority and majority carrier mobility for a wide range of temperatures and injection levels, and thus characterize and simulate the behavior of solar cells or other devices under various conditions.

Solar-grade silicon often contains high concentrations of both acceptors and donors. Such dopant compensation reduces the net doping $\left(p_{0}=N_{A}-N_{D}\right.$ for $p$-type silicon) and thus increases the resistivity $\left(\rho=1 / q p_{0} \mu_{P}\right)$. For the same resistivity, a compensated sample will, therefore, have more ionized impurities than a noncompensated sample. This greater ionized impurity scattering can be expected to reduce the mobility in the compensated sample. Previous measurements using free carrier absorption, ${ }^{10}$ capacitance voltage,${ }^{11-13}$ glow discharge mass spectroscopy, ${ }^{14}$ or the Hall method $^{11,15,16}$ have shown that the majority carrier mobility in silicon is strongly affected by compensation. Other measurements in compensated germanium have shown a similar trend for majority carriers ${ }^{17,18}$ and minority carriers. ${ }^{19}$

\footnotetext{
a) Author to whom correspondence should be addressed. Electronic mail: fiacre.rougieux@anu.edu.au.
}

The increased impurity concentration and the different scattering properties of ionized acceptors and donors are only two of the peculiarities that make carrier mobilities in compensated silicon intriguing. Another interesting aspect is that the effect of compensation is not the same for electron and holes. In $p$-type compensated silicon, even though both electrons and holes will be scattered by more impurities $\left(N_{A}\right.$ and $N_{D}$ ), they will also see fewer free holes. The lower hole concentration will result in lower hole-hole screening and lower electron-hole scattering. This means that while the higher impurity concentration reduces the electron mobility, the lower free hole concentration will tend to increase the electron mobility. The problem of mobility in compensated silicon is thus not straightforward, and experimental data for both majority and minority carrier mobility are needed to ascertain the degree of impact of the various scattering mechanisms.

In this paper, the majority hole conductivity mobility, which is essential to deduce the bulk net doping $p_{0}$ from resistivity $\rho$ measurements, is measured for different acceptor and donor concentrations.

As solar cells are minority carrier devices, it is also of fundamental importance to assess the minority carrier mobility in compensated silicon. Diffusion lengths have been measured in compensated silicon before ${ }^{14,16,20,21}$ but until now, no direct experimental data concerning minority electron mobility in compensated $p$-type silicon has been measured. Such data are presented here.

A common method for measuring the majority carrier mobility is the Hall method. Unfortunately, the Hall method requires knowledge of the Hall factor in order to convert the Hall mobility into the conductivity mobility, which is the parameter of interest for solar cell operation. The Hall factor has been measured before in lightly doped silicon highly 
TABLE I. Measured and modeled (Klaassen) minority and majority carrier mobility in compensated and noncompensated silicon.

\begin{tabular}{|c|c|c|c|c|c|c|c|c|}
\hline \multirow{3}{*}{$\begin{array}{c}\rho \\
(\Omega \mathrm{cm})\end{array}$} & \multirow{2}{*}{\multicolumn{3}{|c|}{$\begin{array}{l}\text { Concentration } \\
\left(\mathrm{cm}^{-3}\right)\end{array}$}} & \multicolumn{3}{|c|}{$\begin{array}{l}\text { Measured mobility } \\
\quad\left(\mathrm{cm}^{2} \mathrm{~V}^{-1} \mathrm{~s}^{-1}\right)\end{array}$} & \multicolumn{2}{|c|}{$\begin{array}{l}\text { Klaassen mobility } \\
\left(\mathrm{cm}^{2} \mathrm{~V}^{-1} \mathrm{~s}^{-1}\right)\end{array}$} \\
\hline & & & & \multicolumn{2}{|r|}{ Majority } & \multirow[b]{2}{*}{ Minority } & \multirow[b]{2}{*}{ Majority } & \multirow[b]{2}{*}{ Minority } \\
\hline & $N_{A}$ & $N_{D}$ & $p_{0}$ & Hall & Conductivity & & & \\
\hline \multicolumn{9}{|c|}{$p$-type control } \\
\hline 4.70 & $2.10 \times 10^{15}$ & $\cdots$ & $2.10 \times 10^{15}$ & 316 & $\cdots$ & $\cdots$ & 456 & 1283 \\
\hline 1.28 & $1.15 \times 10^{16}$ & $\cdots$ & $1.15 \times 10^{16}$ & 281 & $435 \pm 13$ & $1120 \pm 100$ & 425 & 1082 \\
\hline 0.48 & $3.50 \times 10^{16}$ & $\cdots$ & $3.50 \times 10^{16}$ & 246 & $372 \pm 11$ & $765 \pm 80$ & 382 & 878 \\
\hline \multicolumn{9}{|c|}{$p$-type compensated } \\
\hline 1.14 & $4.00 \times 10^{16}$ & $2.5 \times 10^{16}$ & $1.50 \times 10^{16}$ & 244 & $365 \pm 11$ & $660 \pm 57$ & 358 & 838 \\
\hline 0.53 & $8.10 \times 10^{16}$ & $4.05 \times 10^{16}$ & $4.05 \times 10^{16}$ & 218 & $291 \pm 9$ & $476 \pm 87$ & 315 & 684 \\
\hline
\end{tabular}

compensated with thermal donors. ${ }^{11}$ In our study, the Hall factor is determined by a direct comparison between independent measurements of the Hall mobility and conductivity mobility on the same samples (boron and phosphorus doped).

Finally, it is also of interest for further simulation and characterization to know if conventional mobility models such as Klaassen's ${ }^{2,3}$ model can be used safely for compensated silicon. We, therefore, compare Klaassen's model with our experimental results to assess its accuracy.

\section{EXPERIMENTAL METHODS}

The samples used in these studies were 155 $\times 155 \mathrm{~mm}^{2} \quad$ pseudosquare, $\quad p$-type, $\langle 100\rangle$-oriented Czochralski-grown silicon wafers with random pyramid texture. The samples came from three control ingots (noncompensated), which were boron-doped, with samples resistivities of 4.78, 1.28, and $0.48 \Omega \mathrm{cm}$, and from two compensated ingots, doped with both boron and phosphorus, with samples resistivities of 1.14 and $0.43 \Omega \mathrm{cm}$. For more details regarding these samples see Ref. 23. The acceptor concentrations $N_{A}$ were previously determined using a method based on the association time constant of iron-boron pairs. ${ }^{22}$ The donor concentrations $N_{D}$ were determined using net dopant densities measured by electrochemical capacitance voltage (ECV) combined with acceptor density values, via $N_{D}=N_{A}-p_{0}$, and as listed in Table I.

All the samples were surface etched to remove the preexisting random pyramid texture. The samples were cut in $1 \times 1 \mathrm{~cm}^{2}$ pieces using a dicing saw. Aluminum contacts were then evaporated at each corner of the samples according to the van der Pauw structure. ${ }^{23}$ The resistivity $(\rho)$ was measured using a four point probe setup on the four aluminum contacts.

The conductivity carrier concentration $\left(p_{0, C}\right)$ was then measured on the same samples using a CPV21 ECV profiler with contact on the Al pads. The area of the ECV crater was measured externally by a pin profiler (Dektak). This allowed for the correction of the calculated dopant density as explained by Bock et al. ${ }^{24}$ The profile was found to stabilize after $0.1 \mu \mathrm{m}$. To minimize the uncertainty, more than 30 net dopant measurements were made until a depth of 0.4-0.5 $\mu \mathrm{m}$ was reached. The dopant density value was de- termined as the mode of the net dopant values. By avoiding the use of the average value, the initial measurements and the local false measurements (mainly due to bubbles in the electrolyte) were excluded. The conductivity mobility was then calculated $\left(\mu_{C}=1 / q p_{0, C} \rho\right)$.

In order to perform minority carrier mobility measurements with the technique developed by Sproul et al. ${ }^{25}$ further $155 \times 155 \mathrm{~mm}^{2}$ square wafers were cut into $4 \times 4 \mathrm{~cm}^{2}$ pieces. The wafers were then etched to different thicknesses ranging from 90 to $170 \mu \mathrm{m}$ using a standard $\mathrm{HNO}_{3}: \mathrm{HF}$ etch. This tends to yield non uniform surface thickness near the edges. Nevertheless the $2 \times 2 \mathrm{~cm}^{2}$ central part was found to be uniform in thickness. The surfaces were next abraded to yield infinite surface recombination velocities. The effective lifetimes were then dominated by surface recombination, and their magnitudes determined by the diffusivity (or mobility) of minority carriers, as described below.

These lifetimes were measured using the microwave photoconductance decay technique with an excitation wavelength of $904 \mathrm{~nm}$. This is the same wavelength used by Sproul et $a .^{25}$ and in these thin wafers will generate almost flat carrier profiles. The lifetime variation due to increasing laser power was monitored in order to achieve a high signal/ noise ratio without reaching high-injection conditions, which would affect the mobility. As we observed no discernable variation in the effective lifetime with intermediate laser power and a high signal/noise ratio, such laser power was used. The lifetime was measured more than 25 times with averaging over 1024 pulses to get a low standard deviation. Figure 1 shows the reduction in the measured effective lifetime with thickness, plotted in the manner used by Sproul, allowing the minority carrier electron mobility to be determined on each of the samples. The uncertainty in the mobilities was estimated using a best fit within the error bars of the $1 / \tau$ versus $(\pi / W)^{2}$ data (with $W$ the sample thickness).

The Hall carrier concentration $\left(p_{0, H}\right)$ was then measured on the van der Pauw structure using an Accent HL5500PC Hall Effect measurement system with a magnetic field strength of $0.32 \mathrm{~T}$ (low magnetic field). This allowed for the determination of the Hall mobility $\left(\mu_{H}=1 / q p_{0, H} \rho\right)$. Using both Hall and conductivity measurements allows us to determine the Hall mobility and the conductivity mobility, and, therefore, the Hall factor. By making both carrier concentra- 


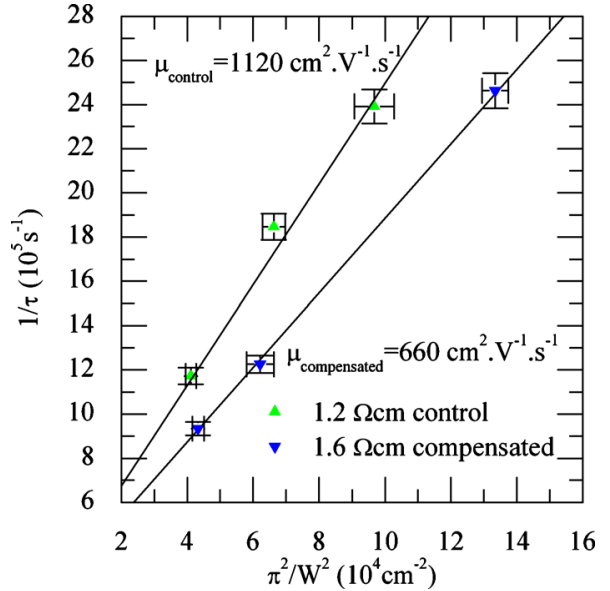

FIG. 1. (Color online) Plot of $1 / \tau$ vs $(\pi / W)^{2}$ to determine the minority carrier mobility. The error in the mobility is determined using the best fit within the error bars.

tion measurements at the same place on the same sample, we avoid any uncertainty due to dopant differences between samples.

\section{RESULTS AND DISCUSSION}

\section{A. Majority carrier conductivity mobility}

The majority hole conductivity mobilities determined by ECV and resistivity measurements are listed in Table I for two control samples and the two compensated samples. Note that these four samples occur in two pairs with similar values of $p_{0}$, which provides a useful basis for comparison. The results reveal a $15 \%-20 \%$ reduction in the hole mobility in the compensated silicon wafers compared to noncompensated silicon samples with similar net doping. For both the controls and the compensated samples the measured values are in very good agreement with those predicted by Klaassen's model.

The results are also plotted in Fig. 2, as a function of the acceptor concentration $N_{A}$. Klaassen's model is shown for three cases, with $N_{D}=0 \mathrm{~cm}^{-3}, N_{D}=2.5 \times 10^{16} \mathrm{~cm}^{-3}$, and $N_{D}=4.05 \times 10^{16} \mathrm{~cm}^{-3}$. The $N_{D}=0 \mathrm{~cm}^{-3}$ case corresponds to

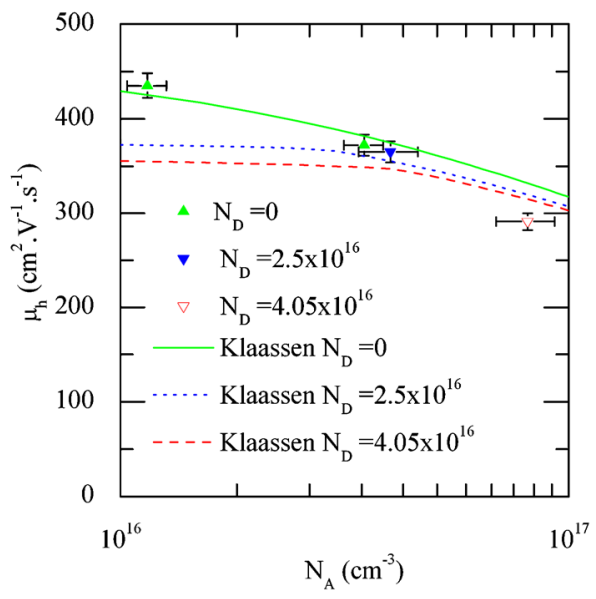

FIG. 2. (Color online) Majority conductivity hole mobility in compensated and noncompensated $p$-type silicon vs acceptor concentration for different donor concentrations.

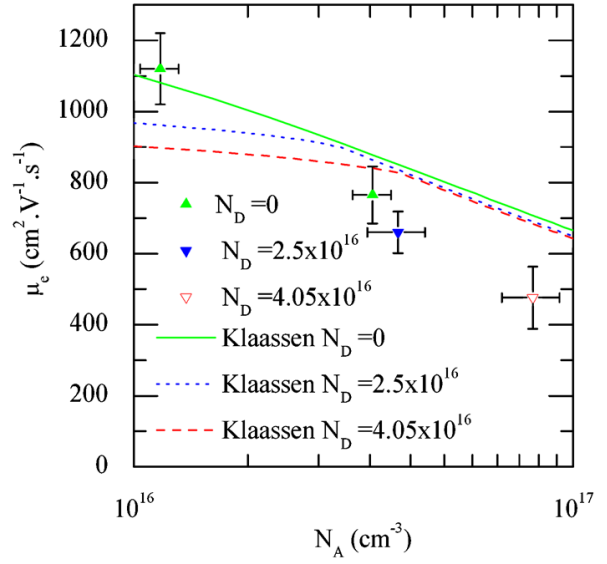

FIG. 3. (Color online) Minority electron mobility in compensated and noncompensated $p$-type silicon vs acceptor concentration for different donor concentrations.

the two control wafers, while the other two curves relate to the two compensated samples. The plot again reveals the good agreement with Klaassen's model.

\section{B. Minority carrier conductivity mobility}

The minority carrier mobility was measured using the Sproul method on samples with mechanically abraded surfaces. ${ }^{25}$ This method only requires the knowledge of two quantities, the effective lifetime $\tau$ and the thickness $W$ of the sample. Due to the very high surface recombination velocity the lifetime is dominated in low injection by the diffusion rate of the minority carriers to the surface. For the case of an infinite surface recombination velocity, the relation between the effective lifetime $\left(\tau_{e f f}\right)$, the diffusion coefficient $\left(D_{n}\right)$, the sample thickness $(W)$ and bulk lifetime $\left(\tau_{b}\right)$ is ${ }^{25}$

$$
\frac{1}{\tau_{\text {eff }}}=\frac{1}{\tau_{b}}+\left(\frac{\pi}{W}\right)^{2} D_{n} .
$$

Knowing the thickness and the effective lifetime one can, therefore, deduce the electron diffusion coefficient. Because some of our samples have quite low resistivity, with low bulk lifetime, the diffusion constant was determined from the slope of a plot of $1 / \tau$ versus $(\pi / W)^{2}$ using samples of different thickness, as explained by Sproul et al. ${ }^{25}$ This allows the impact of the bulk lifetime to be eliminated from the analysis. The fit for a compensated sample of resistivity $1.6 \Omega \mathrm{cm}$ and noncompensated sample of resistivity $1.2 \Omega \mathrm{cm}$ is shown in Fig. 1. The resulting electron mobilities are listed in Table I. There is a significant 35\%-45\% reduction in electron mobility in our compensated silicon samples compared to the noncompensated silicon samples with similar net doping.

Figure 3 shows the minority electron mobilities plotted as a function of $N_{A}$, in analogy to Fig. 2. While the control samples are in reasonable agreement with Klaassen's model, the compensated samples lie well below the curves predicted by it. 


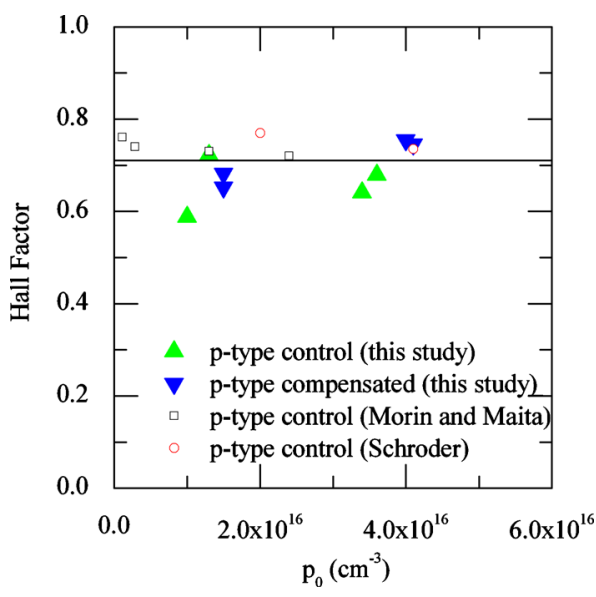

FIG. 4. (Color online) Measured Hall factor in noncompensated and compensated silicon compared to noncompensated Hall factors from the literature. The straight line represents the average value of 0.71 for the Hall factor in compensated silicon.

\section{Hall factor in compensated silicon}

The Hall factor depends on the carrier type, the dominant scattering mechanisms, temperature and the magnitude of the magnetic field. The Hall factor approaches unity when the magnetic field is strong. ${ }^{26}$ However, for practical reasons it is often difficult to measure under such high magnetic fields. Therefore, it is of fundamental importance to measure the Hall factor $r_{H}$ in compensated silicon in order to be able to convert low-field Hall mobilities into conductivity mobilities. Moreover, the Hall factor is larger than 1 in $n$-type silicon and smaller than 1 in $p$-type silicon. ${ }^{26}$ This raises uncertainty about the value of the Hall factor when both dopant types are present in the bulk, as is the case for compensated silicon.

The relationship between the Hall mobility $\mu_{H}$ and the conductivity mobility $\mu_{C}$ is expressed as $\mu_{H}=r_{H} \mu_{C}$. The resistivity has no part in the determination of the Hall factor, and thus the Hall factor can also be expressed as the ratio of the conductivity carrier density $p_{0, C}$ over the Hall carrier density $p_{0, H}$ as follows: $r_{H}=p_{0, C} / p_{0, H}$. Using the ECV and Hall carrier concentrations measured in this work, the Hall factor at room temperature was thus determined.

Figure 4 shows that the Hall factor at room temperature is approximately constant in the $10^{16}-10^{17} \mathrm{~cm}^{-3}$ dopant range. An average value of 0.71 is a good approximation for both control and compensated samples. The values of the Hall factor in the control samples are close to those found in the literature for $p$-type silicon. ${ }^{27,28}$ In compensated silicon, the Hall factor is close to the value of 0.74 estimated by Libal et al. ${ }^{15}$ using Hall and simulated conductivity mobility data. It is also in agreement with the $0.85 \pm 0.15$ Hall coefficient measured in $p$-type silicon compensated by thermal donors. ${ }^{11}$

Table I shows the measured Hall and conductivity majority mobilities. Using a unity Hall factor would lead to approximately a $30 \%$ error in the determination of the conductivity mobility, which is quite significant.

\section{Discussion}

The measured conductivity mobilities were compared to Klaassen's mobility model. Klaassen's model is the standard low field mobility model used in semiconductor simulations near room temperature. It has the advantage of taking into account acceptor and donor scattering mechanisms separately, and, therefore, has the potential to properly account for scattering mechanisms in compensated material of different compensation ratios and dopant concentrations. The model inputs, namely the acceptor and donor concentrations, are shown in Table I.

Within experimental error, Klaassen's mobility model gives very good agreement with our measurements for the majority hole mobility in compensated $p$-type silicon. Combined with an accurate model of the bulk dopant using known segregation coefficients for the dopant species present, ${ }^{29}$ this allows for the conversion of resistivity measurements into net dopant densities in compensated ingots.

However, the measured minority electron mobilities are, even considering the uncertainties (see Fig. 3), significantly lower than predicted by Klaassen's model for the two compensated samples (by about 15\% and 20\%).

Klaassen's mobility model uses the data of Masetti et al. ${ }^{4}$ in noncompensated silicon to identify the main scattering mechanisms. The discrimination between impurity and electron-hole scattering is made using theoretical calculations fitted to noncompensated data. The amount of scattering due to these mechanisms is different in compensated silicon, with less majority carrier screening and electron scattering but higher impurity screening and impurity scattering. If not weighted properly, the altered electron-hole scattering could lead to the discrepancy observed between our experimental electron mobility data and Klaassen's model.

Our results also have implications for another current question regarding compensated silicon, namely, whether B-P pairs exist or not. ${ }^{21,30}$ If B-P pairs were present in the material they would act as single scattering centers for both holes and electrons. The resulting density of ionized dopants causing scattering would thus be expected to be lower. However, the fact that we observe a good agreement with Klaassen's model using the total dopant concentrations $N_{A}$ and $N_{D}$ as inputs indicates that B-P pairs are not present to any significant degree, in agreement with other recent studies. ${ }^{10,31}$

\section{CONCLUSION}

We have shown that both the minority and majority conductivity mobilities are strongly affected by dopant compensation in silicon. In particular, minority carrier mobilities are more affected than majority carrier mobilities. For similar resistivities, the majority hole mobility is reduced by around $20 \%$ in the samples studied here, while the minority electron mobility is reduced by around $40 \%$. This reduction in carrier mobility would, in isolation, have a negative impact on the solar cell efficiency, with minority carrier diffusion lengths reduced by around $22 \%$ due to compensation. Nevertheless, the diffusion length also depends on the carrier lifetime, which is known to increase with compensation, ${ }^{14,15}$ and 
which may offset the reduction in mobility. We have found the Hall factor at room temperatures to be similar in compensated and noncompensated silicon, with a value of approximately 0.71 . Contrary to previous conjectures, ${ }^{15}$ the decrease in the Hall mobility in compensated silicon cannot be explained by an increase in the Hall factor. We also conclude that Klaassen's mobility model can be used to accurately model majority carrier mobilities in compensated silicon but appears to overestimate the minority carrier mobility.

\section{ACKNOWLEDGMENTS}

This work was supported by the Australian Research Council (ARC) and by the DAAD/Go8 researcher exchange funding scheme. We are grateful to Kai Petter of Q-cells and Bart Geerligs of ECN for kindly supplying the wafers used in this study. Thanks are also due to Chris Samundsett for assisting with sample preparation and to Martin Wolf for helping with the ECV measurements.

${ }^{1}$ S. Reggiani, M. Valdinoci, L. Colalongo, M. Rudan, G. Baccarani, A. D. Stricker, F. Illien, N. Felber, W. Fichtner, and L. Zullino, IEEE Trans. Electron Devices 49, 490 (2002).

${ }^{2}$ D. B. M. Klaassen, Solid-State Electron. 35, 961 (1992).

${ }^{3}$ D. B. M. Klaassen, Solid-State Electron. 35, 953 (1992).

${ }^{4}$ G. Masetti, M. Severi, and S. Solmi, IEEE Trans. Electron Devices 30, 764 (1983).

${ }^{5}$ N. D. Arora, J. R. Hauser, and D. J. Roulston, IEEE Trans. Electron Devices 29, 292 (1982).

${ }^{6}$ J. M. Dorkel and P. Leturcq, Solid-State Electron. 24, 821 (1981).

${ }^{7}$ W. R. Thurber, R. L. Mattis, Y. M. Liu, and J. J. Filliben, J. Electrochem. Soc. 127, 2291 (1980).

${ }^{8}$ W. R. Thurber, R. L. Mattis, Y. M. Liu, and J. J. Filliben, J. Electrochem. Soc. 127, 1807 (1980).

${ }^{9}$ D. M. Caughey and R. E. Thomas, Proc. IEEE 55, 2192 (1967).

${ }^{10}$ D. Macdonald, F. Rougieux, A. Cuevas, B. Lim, J. Schmidt, M. Di Sabatino, and L. J. Geerligs, J. Appl. Phys. 105, 093704 (2009).
${ }^{11}$ J. Veirman, S. Dubois, N. Enjalbert, J. P. Garandet, D. R. Heslinga, and M. Lemiti, Solid-State Electron. 54, 671 (2010).

${ }^{12}$ B. Lim, A. Liu, D. Macdonald, K. Bothe, and J. Schmidt, Appl. Phys. Lett. 95, 232109 (2009).

${ }^{13}$ K. Peter, R. Kopecek, A. Soiland, and E. Enebakk, Proceedings of the 23rd European Photovoltaic Solar Energy Conference, Valencia, Spain (WIP-Renewable Energies, Munich, Germany, 2008).

${ }^{14}$ S. Dubois, N. Enjalbert, and J. P. Garandet, Appl. Phys. Lett. 93, 032114 (2008).

${ }^{15}$ J. Libal, S. Novaglia, M. Acciarri, S. Binetti, R. Petres, J. Arumughan, R. Kopecek, and A. Prokopenko, J. Appl. Phys. 104, 104507 (2008).

${ }^{16}$ S. Pizzini and C. Calligarich, J. Electrochem. Soc. 131, 2128 (1984).

${ }^{17}$ L. M. Falicov and M. Cuevas, Phys. Rev. 164, 1025 (1967).

${ }^{18}$ M. Cuevas, Phys. Rev. 164, 1021 (1967).

${ }^{19}$ M. B. Prince, Phys. Rev. 92, 681 (1953).

${ }^{20}$ J. Kraiem, R. Einhaus, and H. Lauvray, Proceedings of the 34th IEEE Photovoltaic Specialists Conference, Philadelphia, USA (IEEE, New York, 2009).

${ }^{21}$ W. Krühler, C. Moser, F. W. Schulze, and H. Aulich, Proceedings of the Eighth European Photovoltaic Solar Energy Conference, Florence, Italy (Kluwer Academic, Dordrecht, The Netherlands, 1988), p. 1181.

${ }^{22}$ D. Macdonald, A. Cuevas, and L. J. Geerligs, Appl. Phys. Lett. 92, 202119 (2008).

${ }^{23}$ L. J. Van der Pauw, Philips Tech. Rev. 20, 221 (1958).

${ }^{24} \mathrm{R}$. Bock, P. Altermatt, and J. Schmidt, Proceedings of the 23rd European Photovoltaic Solar Energy Conference, Valencia, Spain (WIP-Renewable Energies, Munich, Germany, 2008).

${ }^{25}$ A. B. Sproul, M. A. Green, and A. W. Stephens, J. Appl. Phys. 72, 4161 (1992).

${ }^{26}$ J. F. Lin, S. S. Li, L. C. Linares, and K. W. Teng, Solid-State Electron. 24, 827 (1981).

${ }^{27}$ D. K. Schroder, T. T. Braggins, and H. M. Hobgood, J. Appl. Phys. 49, 5256 (1978).

${ }^{28}$ F. J. Morin and J. P. Maita, Phys. Rev. 96, 28 (1954).

${ }^{29}$ F. Padovani, J. Appl. Phys. 43, 2003 (1972).

${ }^{30} \mathrm{R}$. Kopecek, J. Arumughan, and K. Peter, Proceedings of the 23rd European Photovoltaic Solar Energy Conference, Valencia, Spain (WIPRenewable Energies, Munich, Germany, 2008).

${ }^{31}$ T. Schutz-Kuchly, J. Veirman, S. Dubois, and D. R. Heslinga, Appl. Phys. Lett. 96, 093505 (2010). 